(2) Open Access Full Text Article

\title{
Association between CHFR gene hypermethylation and gastric cancer risk: a meta-analysis
}

This article was published in the following Dove Press journal:

OncoTargets and Therapy

8 December 2016

Number of times this article has been viewed

\author{
Hua Shi \\ Xiaojing Wang \\ jianbo Wang \\ Jundi Pan \\ Junwei Liu \\ Bin Ye
}

Department of Gastroenterology, Lishui Central Hospital, The Fifth Affiliated Hospital of Wenzhou Medical University, Lishui Hospital of Zhejiang University, Lishui, People's Republic of China
Correspondence: Bin Ye Department of Gastroenterology, Lishui Central Hospital, The Fifth Affiliated Hospital of Wenzhou Medical University, Lishui Hospital of Zhejiang University, 289 Kuocang Road, Lishui, Zhejiang 323000 , People's Republic of China

Tel +86578228 6237

Fax +86578213 3457

Email binye.Ish@gmail.com
Background: The association between the hypermethylation of $C H F R$ gene and gastric cancer risk has been investigated by a number of studies. However, the sample size of the majority of these studies was very small. To get a more a convincing conclusion, here we performed a meta-analysis of the previously published studies to assess the association between $C H F R$ methylation and the risk of gastric cancer.

Methods: Eligible studies were identified by searching the MEDLINE/PubMed, Embase, and Web of Science databases before May 2016 without any language restriction. The strength of the association was estimated by odds ratio with its $95 \%$ confidence interval (CI).

Results: Totally 1,399 samples, including 758 gastric cancer cases and 641 controls, from 13 studies were included in the present meta-analysis. Compared with non-cancer controls, the pooled OR of CHFR methylation in gastric cancer patients was 9.08 (95\% CI: 6.40-12.88, $P<0.001$ ), suggesting that the methylation of $C H F R$ was significantly associated with increased risk of gastric cancer. Similar results were observed when subgroup analyses were performed stratified by country, ethnicity, and methylation testing methods.

Conclusion: Our meta-analysis showed a strong positive correlation between CHFR methylation and risk of gastric cancer, suggesting that $C H F R$ methylation might be a promising biomarker for the diagnosis of gastric cancer.

Keywords: $C H F R$, methylation, tumor suppressor gene, gastric cancer, risk

\section{Introduction}

Gastric cancer is one of the most commonly diagnosed human cancers, and it is among the leading causes of cancer-related death worldwide. ${ }^{1}$ More than $70 \%$ of new gastric cancer cases and deaths occur in developing countries. The incidence rate of gastric cancer is high in Eastern Asia, Central and Eastern Europe, and South America, but low in Northern America and Africa. ${ }^{2}$ It is well established that chronic infection with Helicobacter pylori is the most common risk factor for gastric cancer, since about $90 \%$ of new noncardia gastric cancer cases worldwide attributed to this bacteria. . $^{3,4}$ In addition to bacteria infection, genetic and epigenetic changes of some oncogenes and tumor suppressor genes (TSG) have been involved in the initiation and development of gastric cancer., 5

The CHFR gene is localized to chromosome $12 \mathrm{q} 24.33$, and it was identified as a cell-cycle checkpoint gene. ${ }^{7,8}$ In response to mitotic stress induced by microtubule inhibitors, the $C H F R$ protein causes a delay in chromosome condensation and entry into metaphase. However, cancer cells lacking $C H F R$ entered metaphase without delay. ${ }^{8}$ The $C H F R$ protein possesses an N-terminal forkhead-associated (FHA) domain, a central RING finger (RF) domain, and a C-terminal cysteine-rich 
(CR) region. The FHA and CR regions are essential for its checkpoint function, and the RF domain is required for the ubiquitin ligase activity of CHFR. ${ }^{9}$ CHFR is widely expressed in normal tissues, and loss or reduced expression of $C H F R$ has been reported in several primary tumors. Interestingly, in cancer cell lines and several types of primary cancer, the decreased $C H F R$ expression was reported to be caused by the hypermethylation of the $\mathrm{CpG}$ island in the promoter region of this gene, ${ }^{10-12}$ including gastric cancer. ${ }^{13,14}$

Ever since the initial report of hypermethylation of CHFR in gastric cancer, ${ }^{15}$ a growing number of studies have investigated the association of CHFR methylation and risk of gastric cancer. However, the sample size of these studies was very small; most of them enrolled less than 100 cancer cases. Based on the notion that the statistical power is low when there is only a small number of cases enrolled in a case-control study, therefore, we conducted a meta-analysis of the previously published studies to assess whether there is an association between CHFR methylation and risk of gastric cancer.

\section{Methods}

\section{Literature search strategy}

The MEDLINE/PubMed, Embase, and Web of Science databases were used for searching literatures. The search was carried out before May 2016 without any language restriction. The keywords used for paper searching were CHFR, methylation, stomach, gastric, and cancer. To search for additional relevant publications, the reference lists from relevant primary studies and review articles were also checked manually.

\section{Study selection and data extraction}

We selected studies if they met all of the following criteria: 1) the study had a case-control design; 2) the study focused on the relationship between $C H F R$ hypermethylation and risk of gastric cancer; 3 ) the frequency of the CHFR methylation status had been reported or could be calculated; and 4) if several studies had overlapping cancer or control cases, the studies with the largest sample size were selected in the present study.

The following information were extracted, respectively, by two investigators: last name of the first author, year of the publication, country where study conducted, subject ethnicity, testing materials, numbers of cases and controls, and the method for methylation testing in each study. The two investigators reached a consensus on all items.

\section{Statistical analyses}

The strength of the association between CHFR methylation and gastric cancer risk was assessed by odds ratio (OR) with its $95 \%$ confidence interval (CI). The heterogeneity among the studies was estimated by a chi-square-based $Q$-test and further quantified by the $I^{2}$ metric. ${ }^{16}$ The fixed-effects model was selected to calculate the pooled OR when the betweenstudy heterogeneity was absent. ${ }^{17}$ Otherwise, the randomeffects model was selected. ${ }^{18}$ Begg's funnel plots and Egger's linear regression test were used to examine whether the results were affected by publication bias. ${ }^{19}$ If publication bias was observed, the nonparametric "trim and fill" method was carried out for estimating the effect of missing studies on the overall outcome. ${ }^{20}$ Moreover, subgroup analyses were also performed stratified by country, ethnicity, and methylation testing methods, respectively. All of the statistical analyses were carried out by the Stata software (version 10; Stata Corp, College Station, TX, USA). All the $P$-values were two-sided, and $P<0.05$ was considered to be statistically significant.

\section{Results}

\section{Characteristics of included studies}

According to the literature search strategy and selection criteria, 13 independent articles were eventually included in the present meta-analysis..$^{13-15,21-30}$ The characteristics of all the included studies are summarized in Table 1 . The 13 studies were published between 2003 and 2016, and all of them were written in English. Among the 13 studies, eight studies came from investigations involving Japanese populations, three came from China, one came from Korea, and one came from the USA. For all the enrolled studies, the gastric cancer samples were all obtained from gastric cancer tissues, and the controls were all from corresponding non-neoplastic gastric mucosa. Seven of the 13 studies used methylation-specific PCR (MSP) to detect CHFR methylation status in gastric cancer and control samples, two studies used bisulfite treatment and combined bisulfite restriction analysis (COBRA), and four studies used other methylation detection methods. Totally, 1,399 samples, including 758 gastric cancer cases and 641 controls, were involved in the present meta-analysis.

\section{Quantitative data analysis}

The between-study heterogeneity of all the 13 studies included in the present study was firstly analyzed, and no significant heterogeneity among them was found $(P=0.172$, $I^{2}=27.0 \%$, Figure 1, Table 2). Therefore, the strength of the association between methylation of CHFR and risk of 
Table I Characteristics of studies included in the present meta-analysis

\begin{tabular}{|c|c|c|c|c|c|c|c|}
\hline Author & Year & Country & Ethnicity & Materials & Cases (n) & Controls (n) & Testing methods \\
\hline Satoh et al ${ }^{15}$ & 2003 & Japan & Asian & Tissue & 61 & 44 & COBRA \\
\hline Honda et $\mathrm{al}^{23}$ & 2004 & Japan & Asian & Tissue & 71 & 71 & MSP \\
\hline Kang et $\mathrm{a}^{24}$ & 2004 & Korea & Asian & Tissue & 43 & 14 & Bisulfite PCR and sequencing \\
\hline Homma et $\mathrm{al}^{22}$ & 2005 & Japan & Asian & Tissue & 52 & 52 & MSP \\
\hline Koga et $\mathrm{al}^{25}$ & 2006 & Japan & Asian & Tissue & 46 & 46 & MSP \\
\hline Morioka et $\mathrm{al}^{26}$ & 2006 & Japan & Asian & Tissue & 53 & 53 & MSP \\
\hline Yoshida et $\mathrm{al}^{30}$ & 2006 & Japan & Asian & Tissue & 41 & 41 & COBRA \\
\hline Gao et $\mathrm{al}^{13}$ & 2008 & China & Asian & Tissue & 20 & 20 & MSP \\
\hline Oki et $\mathrm{a}^{27}$ & 2009 & Japan & Asian & Tissue & 59 & 59 & MSP \\
\hline Hiraki et $\mathrm{a}^{21}$ & 2010 & Japan & Asian & Tissue & 49 & 49 & qMSP \\
\hline Hu et al ${ }^{14}$ & 2011 & China & Asian & Tissue & 123 & 123 & MSP \\
\hline Wang et $\mathrm{al}^{29}$ & 2014 & China & Asian & Tissue & 117 & 46 & MethyLight \\
\hline Sepulveda et $\mathrm{a}^{28}$ & 2016 & USA & Caucasian & Tissue & 23 & 23 & Next-generation sequencing \\
\hline
\end{tabular}

Abbreviations: COBRA, combined bisulfite restriction analysis; MSP, methylation-specific PCR; qMSP, quantitative methylation-specific PCR; PCR, polymerase chain reaction.

gastric cancer was determined by the fixed-effects model.

Overall, compared with non-cancer controls, the pooled OR of CHFR methylation in gastric cancer patients was 9.08 (95\% CI: 6.40-12.88, $P<0.001$, Figure 1, Table 2), suggesting that $C H F R$ methylation was associated with an increased risk of gastric cancer.

We next performed subgroup analyses stratified by country, ethnicity, and methylation testing methods, respectively. Country-specific OR showed an increased risk for individuals carrying the methylated $C H F R$ compared with those without $C H F R$ gene methylation in Japan (OR=9.29, 95\% CI: $6.00-14.39, P<0.001)$ and China $(\mathrm{OR}=8.30,95 \%$ CI: 4.48-15.40, $P<0.001$, Table 2 ). When combining the studies regarding Japanese, Chinese and Korean together, a strong association between CHFR methylation and gastric cancer risk was found in Asian populations ( $\mathrm{OR}=9.17,95 \%$ CI: 6.44-13.07, $P<0.001$, Table 2). In the stratified analysis by testing methods, significantly increased risks were found in $\operatorname{MSP}(\mathrm{OR}=8.02,95 \%$ CI: 5.29-12.16, $P<0.001)$ and COBRA (OR=53.60, 95\% CI: 7.14-402.38, $P<0.001$, Table 2).

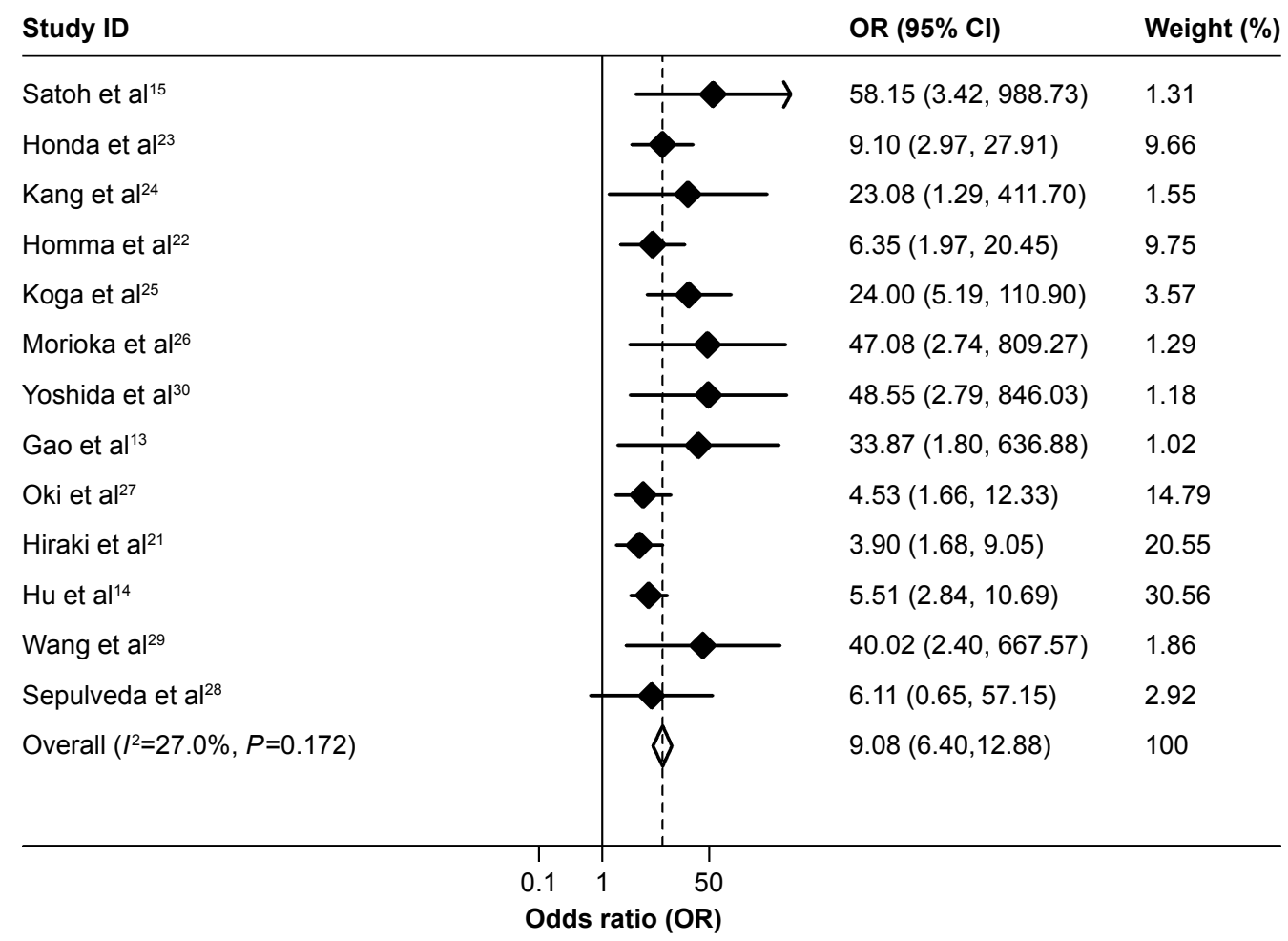

Figure I Forest plots of the association between CHFR methylation and gastric cancer risk. Abbreviations: $\mathrm{Cl}$, confidence interval; $\mathrm{OR}$, odds ratio. 
Table 2 Summary of the association between hypermethylation of CHFR and gastric cancer risk

\begin{tabular}{|c|c|c|c|c|c|c|}
\hline Variables & Study no & Cases/controls & OR $(95 \% \mathrm{CI})$ & $P$-value & $\boldsymbol{P}_{\mathrm{H}} *$ & $I^{2}(\%)$ \\
\hline Total & 13 & $758 / 64 I$ & $9.08(6.40-12.88)$ & $<0.001$ & 0.172 & 27.0 \\
\hline \multicolumn{7}{|l|}{ Country } \\
\hline Japan & 8 & $432 / 415$ & $9.29(6.00-14.39)$ & $<0.001$ & 0.098 & 42.1 \\
\hline China & 3 & $260 / 189$ & $8.30(4.48-15.40)$ & $<0.001$ & 0.170 & 43.7 \\
\hline \multicolumn{7}{|l|}{ Ethnicities } \\
\hline Asian & 12 & $735 / 618$ & $9.17(6.44-13.07)$ & $<0.001$ & 0.125 & 33.1 \\
\hline \multicolumn{7}{|c|}{ Testing methods } \\
\hline MSP & 7 & $424 / 424$ & $8.02(5.29-12.16)$ & $<0.001$ & 0.315 & I5. I \\
\hline COBRA & 2 & $102 / 85$ & $53.60(7.14-402.38)$ & $<0.001$ & 0.930 & 0.0 \\
\hline Others & 4 & $232 / 132$ & $7.75(3.83-15.72)$ & $<0.001$ & 0.216 & 32.8 \\
\hline
\end{tabular}

Note: $* P$-value from the $Q$-test for heterogeneity.

Abbreviations: OR, odds ratio; Cl, confidence interval; MSP, methylation-specific PCR; COBRA, combined bisulfite restriction analysis; PCR, polymerase chain reaction.

\section{Publication bias}

The shape of the funnel plots showed asymmetry in the overall analysis (Figure 2A), meanwhile the results from Egger's test provided statistical evidence for funnel plot asymmetry $(P<0.001)$, indicating the existence of publication bias. To adjust publication bias, we carried out a nonparametric trim and fill method to estimate potential missing studies and assess the effect that these studies might have had on the outcome. As a result, five missing studies were added to the dataset, and the filled dataset showed no evidence of publication bias (Figure 2B). The new dataset moved the estimated pooled OR from 9.68 (95\% CI: 6.40-12.88) to 6.04 (95\% CI: 4.26-8.57). The correction for publication bias did not change the overall interpretation of the dataset, indicating that the strong association between CHFR methylation and gastric cancer risk was statistically robust and reliable.

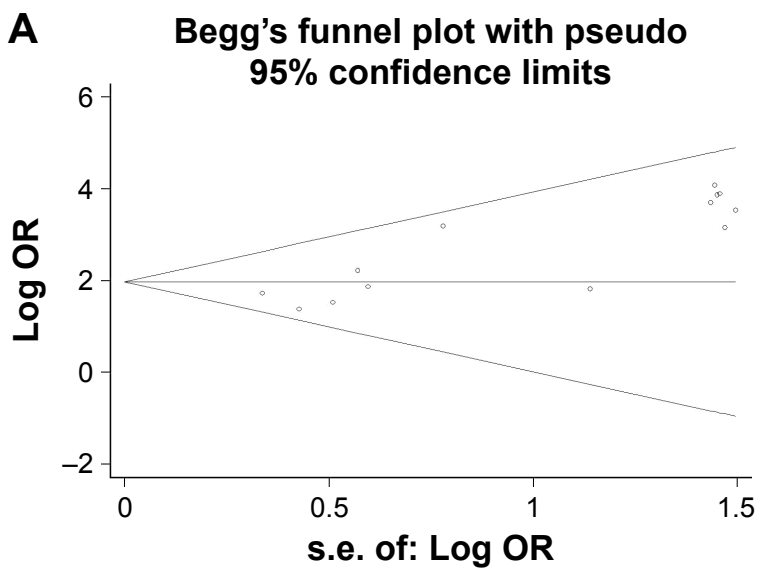

\section{Discussion}

The development of gastric cancer involves genetic or epigenetic alterations that lead to the functional loss of critical genes such as TSG, DNA repair genes, or cell-cycle checkpoint genes. ${ }^{31}$ Increasing number of cancer-related genes have been reported to be methylated in CpG islands of genes' promoter regions. Such type of epigenetic change results in the inactivation of $T S G$ and plays a key role in the epigenetically mediated loss-of-gene function. ${ }^{32}$ Actually, aberrant DNA methylation in the promoter regions of $T S G$ is the most well-defined epigenetic hallmark in gastric cancer. ${ }^{33}$

In recent years, aberrant methylation of the checkpoint gene CHFR associated with gene silencing has been identified in several cancer types, including gastric cancer. ${ }^{10-12,15}$ Based on the studies on CHFR methylation and gastric cancer, we focused on the correlation between CHFR hypermethylation and risk of gastric cancer in the present study. To the best of

\section{B Filled funnel plot with pseudo}

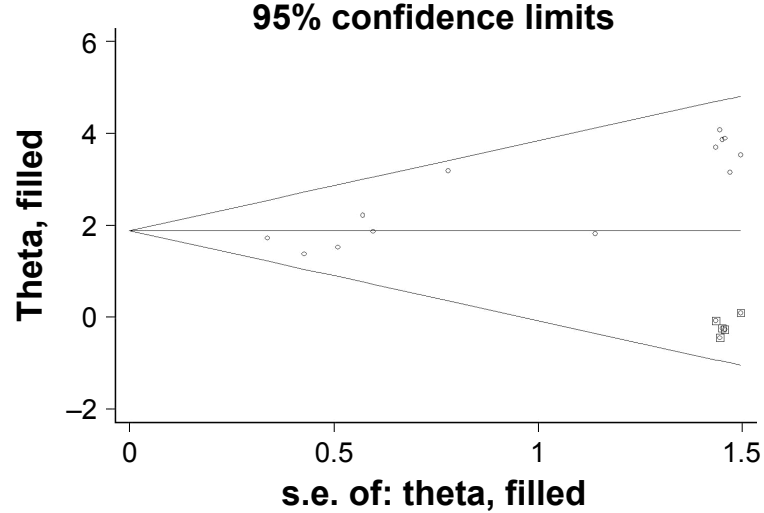

Figure 2 Begg's funnel plot of publication biases. Each point represents a separate study. (A) Begg's funnel plot of publication bias test. (B) Begg's funnel plot of publication bias test after trim and fill method.

Abbreviations: s.e., standard error; OR, odds ratio. 
our knowledge, this is the first meta-analysis study to assess the association of CHFR methylation with gastric cancer risk. Our analysis, combining 13 studies with 758 cancer cases and 641 control samples, revealed that the hypermethylation of CHFR increased the risk of gastric cancer. Particularly, the overall OR for CHFR methylation status in gastric cancer vs non-cancer samples was 9.08 (95\% CI: 6.40-12.88), suggesting a strong association of CHFR methylation with the risk of gastric cancer.

As gastric cancer has a high prevalent rate in Asia, particularly in Japan and China, ${ }^{2}$ we summarized the effect of CHFR methylation on gastric cancer risk in these two countries, and as a result we found that CHFR methylation increased the risk of gastric cancer in both of the two countries. When we pooled the data from 12 studies on Asian populations together, a strong positive association was also observed. In addition to studies from Asian populations, one study came from the Caucasian population. The results of this study showed that the CHFR methylation was significantly increased in gastric cancer compared with non-metaplastic mucosa. ${ }^{28}$ Taken together, these data showed a parallel effect of CHFR methylation on gastric cancer risk among different ethnicities.

The advances in diagnostics and therapeutics in recent years have prolonged survival for gastric cancer patients diagnosed at the early stage. Unfortunately, the overall prognosis is very poor because most of the gastric cancer patients are diagnosed at the advanced stage. ${ }^{34}$ Therefore, an urgent task for gastric cancer research is the development of efficient diagnostic approaches to enable early detection. Several studies investigated the correlation between $C H F R$ methylation and clinicopathological factors, ${ }^{13,14,21,23,24,26,27}$ and none of the studies showed an significant association between CHFR methylation and advanced clinicopathological factors, including tumor stage III/IV or lymphnodemetastasis. These results suggest that the CHFR methylation is an early epigenetic event in gastric cancer. To facilitate DNA methylation detection, many powerful molecular techniques have been invented in the past decades. The well-known techniques includes bisulfite sequencing, COBRA, MSP, quantitative methylation-specific PCR, as well as bisulfite pyrosequencing. ${ }^{35}$ In the present study, the association of CHFR methylation with gastric cancer risk has been stratified by methylation testing methods, and we found significant ORs in subgroups with all testing methods. Moreover, the strong association between CHFR methylation and gastric cancer risk shown in this study is consistent with previous findings that CHFR methylation could be used as an diagnostic factor for several other types of cancer, including lung, colorectal, and esophageal cancers. ${ }^{10-12,36}$ Herein, CHFR methylation is likely to be a molecular marker for early detection in gastric cancer as well as in certain other types of cancer.

Notably, aberrant methylation of CHFR had been reported to be associated with the sensitivity of microtubule inhibitors in several cancer cells including gastric cancer cells in vitro. ${ }^{15}$ However, the clinical significance of $C H F R$ methylation and chemosensitivity of microtubule inhibitors (paclitaxel or docetaxel) in gastric cancer patients was investigated only in a few studies, ${ }^{25,29,30}$ and none of the study showed an significant association between $C H F R$ methylation and the sensitivity of paclitaxel, docetaxel, or a combination of docetaxel and S-1. Since all these studies had very limited number of patients, further research using larger number of patients is necessary to clarify whether $C H F R$ methylation correlates with drug response to microtubule inhibitors.

In summary, this meta-analysis showed a strong association between CHFR methylation and risk of gastric cancer. Although further studies with large number of samples are required to confirm it, the findings in the present study suggest $C H F R$ methylation as a promising molecular marker for early detection in gastric cancer.

\section{Disclosure}

The authors report no conflicts of interest in this work.

\section{References}

1. Stadtländer CT, Waterbor JW. Molecular epidemiology, pathogenesis and prevention of gastric cancer. Carcinogenesis. 1999;20(12): 2195-2208.

2. Torre LA, Bray F, Siegel RL, Ferlay J, Lortet-Tieulent J, Jemal A. Global cancer statistics, 2012. CA Cancer J Clin. 2015;65(2):87-108.

3. Uemura N, Okamoto S, Yamamoto S, et al. Helicobacter pylori infection and the development of gastric cancer. N Engl J Med. 2001;345(11): 784-789.

4. Parsonnet J, Friedman GD, Vandersteen DP, et al. Helicobacter pylori infection and the risk of gastric carcinoma. NEngl J Med. 1991;325(16): 1127-1131.

5. González CA, Sala N, Capellá G. Genetic susceptibility and gastric cancer risk. Int J Cancer. 2002;100(3):249-260.

6. Smith MG, Hold GL, Tahara E, El-Omar EM. Cellular and molecular aspects of gastric cancer. World J Gastroenterol. 2006;12(19): 2979-2990.

7. Kang D, Chen J, Wong J, Fang G. The checkpoint protein Chfr is a ligase that ubiquitinates Plk1 and inhibits Cdc2 at the G2 to M transition. J Cell Biol. 2002;156(2):249-259.

8. Scolnick DM, Halazonetis TD. Chfr defines a mitotic stress checkpoint that delays entry into metaphase. Nature. 2000;406(6794):430-435.

9. Chaturvedi P, Sudakin V, Bobiak ML, et al. Chfr regulates a mitotic stress pathway through its RING-finger domain with ubiquitin ligase activity. Cancer Res. 2002;62(6):1797-1801.

10. Toyota M, Sasaki Y, Satoh A, et al. Epigenetic inactivation of CHFR in human tumors. Proc Natl Acad Sci US A. 2003;100(13):7818-7823. 
11. Mizuno K, Osada H, Konishi H, et al. Aberrant hypermethylation of the CHFR prophase checkpoint gene in human lung cancers. Oncogene. 2002;21(15):2328-2333.

12. Shibata Y, Haruki N, Kuwabara Y, et al. Chfr expression is downregulated by $\mathrm{CpG}$ island hypermethylation in esophageal cancer. Carcinogenesis. 2002;23(10):1695-1699.

13. Gao YJ, Xin Y, Zhang JJ, Zhou J. Mechanism and pathobiologic implications of CHFR promoter methylation in gastric carcinoma. World $J$ Gastroenterol. 2008;14(32):5000-5007.

14. Hu SL, Huang DB, Sun YB, et al. Pathobiologic implications of methylation and expression status of Runx 3 and CHFR genes in gastric cancer. Med Oncol. 2011;28(2):447-454.

15. Satoh A, Toyota M, Itoh F, et al. Epigenetic inactivation of CHFR and sensitivity to microtubule inhibitors in gastric cancer. Cancer Res. 2003; 63(24):8606-8613.

16. Higgins JP, Thompson SG, Deeks JJ, Altman DG. Measuring inconsistency in meta-analyses. BMJ. 2003;327(7414):557-560.

17. Mantel N, Haenszel W. Statistical aspects of the analysis of data from retrospective studies of disease. J Natl Cancer Inst. 1959;22(4): 719-748.

18. DerSimonian R, Laird N. Meta-analysis in clinical trials. Control Clin Trials. 1986;7(3):177-188.

19. Egger M, Davey Smith G, Schneider M, Minder C. Bias in meta-analysis detected by a simple, graphical test. BMJ. 1997;315(7109):629-634.

20. Duval S, Tweedie R. Trim and fill: a simple funnel-plot-based method of testing and adjusting for publication bias in meta-analysis. Biometrics. 2000;56(2):455-463.

21. Hiraki M, Kitajima Y, Sato S, et al. Aberrant gene methylation in the lymph nodes provides a possible marker for diagnosing micrometastasis in gastric cancer. Ann Surg Oncol. 2010;17(4):1177-1186.

22. Homma N, Tamura G, Honda T, et al. Hypermethylation of Chfr and hMLH1 in gastric noninvasive and early invasive neoplasias. Virchows Arch. 2005;446(2):120-126.

23. Honda T, Tamura G, Waki T, Kawata S, Nishizuka S, Motoyama T. Promoter hypermethylation of the Chfr gene in neoplastic and nonneoplastic gastric epithelia. Br J Cancer. 2004;90(10):2013-2016.

24. Kang HC, Kim IJ, Park JH, et al. Promoter hypermethylation and silencing of CHFR mitotic stress checkpoint gene in human gastric cancers. Oncol Rep. 2004;12(1):129-133.
25. Koga Y, Kitajima Y, Miyoshi A, Sato K, Sato S, Miyazaki K. The significance of aberrant CHFR methylation for clinical response to microtubule inhibitors in gastric cancer. J Gastroenterol. 2006;41(2): 133-139.

26. Morioka Y, Hibi K, Sakai M, et al. Aberrant methylation of the CHFR gene in digestive tract cancer. Anticancer Res. 2006;26(3A):1791-1795.

27. Oki E, Zhao Y, Yoshida R, et al. Checkpoint with forkhead-associated and ring finger promoter hypermethylation correlates with microsatellite instability in gastric cancer. World J Gastroenterol. 2009;15(20): 2520-2525.

28. Sepulveda JL, Gutierrez-Pajares JL, Luna A, et al. High-definition $\mathrm{CpG}$ methylation of novel genes in gastric carcinogenesis identified by next-generation sequencing. Mod Pathol. 2016;29(2):182-193.

29. Wang M, Shen L, Deng D. Association between CHFR methylation and chemosensitivity of paclitaxel in advanced gastric cancer. Med Oncol. 2014;31(4):907.

30. Yoshida K, Hamai Y, Suzuki T, Sanada Y, Oue N, Yasui W. DNA methylation of CHFR is not a predictor of the response to docetaxel and paclitaxel in advanced and recurrent gastric cancer. Anticancer Res. 2006;26(1A):49-54.

31. Tamura G. Alterations of tumor suppressor and tumor-related genes in the development and progression of gastric cancer. World $J$ Gastroenterol. 2006;12(2):192-198.

32. Das PM, Singal R. DNA methylation and cancer. J Clin Oncol. 2004; 22(22):4632-4642.

33. Qu Y, Dang S, Hou P. Gene methylation in gastric cancer. Clin Chim Acta. 2013;424:53-65.

34. Karimi P, Islami F, Anandasabapathy S, Freedman ND, Kamangar F. Gastric cancer: descriptive epidemiology, risk factors, screening, and prevention. Cancer Epidemiol Biomarkers Prev. 2014;23(5):700-713.

35. Umer M, Herceg Z. Deciphering the epigenetic code: an overview of DNA methylation analysis methods. Antioxid Redox Signal. 2013;18(15): 1972-1986.

36. Derks S, Cleven AH, Melotte V, et al. Emerging evidence for CHFR as a cancer biomarker: from tumor biology to precision medicine. Cancer Metastasis Rev. 2014;33(1):161-171.
OncoTargets and Therapy

\section{Publish your work in this journal}

OncoTargets and Therapy is an international, peer-reviewed, open access journal focusing on the pathological basis of all cancers, potential targets for therapy and treatment protocols employed to improve the management of cancer patients. The journal also focuses on the impact of management programs and new therapeutic agents and protocols on

\section{Dovepress}

patient perspectives such as quality of life, adherence and satisfaction The manuscript management system is completely online and includes a very quick and fair peer-review system, which is all easy to use. Visit http://www.dovepress.com/testimonials.php to read real quotes from published authors. 